\title{
Antimicrobial Sensitivity Testing Using the Kirby-Bauer Disk Diffusion Method; Limited Utility in Ugandan Hospitals
}

Ivan Segawa ${ }^{1}$, Kenneth Ssebambulidde ${ }^{2}$, Daniel Kiiza ${ }^{2}$ and Jackson Mukonzo ${ }^{1}$

${ }^{1}$ Makerere University College of Health Sciences, P.O. Box 7072, Kampala, Uganda;

${ }^{2}$ Infectious Diseases Institute, College of Health Sciences, Makerere University, P.O. Box 22418, Kampala, Uganda.

Corresponding author: Ivan Segawa, B. Pharm, Makerere University College of Health Sciences. P.O. Box 7072, Kampala, Uganda. Email: ivansegy@gmail.com Telephone: +256 (0)775633955

Keywords: Antimicrobial sensitivity testing; antibiotic resistance; Kirby-Bauer; disk diffusion method; Ugandan hospitals 


\section{ABSTRACT}

Background: Antimicrobial resistance (AMR) is a global health threat responsible for increased healthcare costs and mortality. The World Health Organization (WHO) global action plan on AMR recommends antimicrobial sensitivity testing (AST) and surveillance of antibiotic use to address this threat. We conducted a cross-sectional study to determine the utility of AST in three major referral hospitals in Uganda.

Methods: We used abstraction checklists to collect data on AST requests, AST performed, AST turnaround time, and dispensed antibiotics, from in-patient files and laboratory and pharmacy records. Antibiotic data were summarized using proportions. The relationship between dispensed antibiotics and AST antibiotic-disks was analyzed using spearman's rank correlation and simple linear regression.

Results: Of the 607 in-patient files reviewed, AST was requested in 24 (4.0\%), and done in $13(2.1 \%)$. All three hospitals used the Kirby-Bauer disk diffusion method for AST, with a median turnaround time of 5 days (IQR 4-8). While the frequently used AST antibiotic-disks were cotrimoxazole (13.0\%), ampicillin (11.7\%), and ceftriaxone $(10.5 \%)$, the most dispensed antibiotics were metronidazole (30.3\%), amoxicillin (19.6\%) and ceftriaxone (14.8\%). There was a weak correlation $(r=0.313, p=0.120)$ between dispensed antibiotics and AST antibiotic-disks, and AST performance was not associated with antibiotic consumption.

Conclusion: We report an underutilization of AST, inconsistent with the hospital antibiotic consumption, and may be related to the use of the disk diffusion method. We 
recommend alternative faster and better coordination in the procurement of AST diagnostics in Ugandan hospitals.

\section{INTRODUCTION}

Antimicrobial resistance (AMR) is a global health threat that portends a post-antibiotic era. ${ }^{1}$ Globally, AMR causes more than 700,000 deaths annually, which are estimated to increase to 10 million by $20500^{2,3}$ AMR is marked by the emergence of microorganisms with reduced sensitivity to antimicrobial agents to which they were previously susceptible. Consequently, AMR results in increased length of hospital stay which culminates into high treatment costs and lost productivity, and an estimated $2-3.5 \%$ reduction in gross domestic product. $^{3}$

While AMR is globally distributed, its health and economic impacts pose the greatest challenges to resource-limited settings (RLS) that already carry a disproportionate burden of infectious diseases. ${ }^{2,3}$ Furthermore, many health care facilities in the RLS lack basic diagnostics required for antimicrobial sensitivity testing (AST). ${ }^{4,5}$ As a mitigation strategy, WHO adopted a global action plan (GAP) to build the diagnostic capacity for surveillance of antibiotic use and to improve awareness and understanding of AMR. ${ }^{1}$

Despite the interventions proposed in the GAP, the burden of AMR in Uganda, like many RLS remains unknown. In 2015, Uganda had only eight hospital-based laboratories capable of performing AST. ${ }^{5}$ This low diagnostic capacity hinders AMR surveillance in Uganda, yet there are reports of emerging AMR to the commonly dispensed antibiotics. ${ }^{5}$ Whereas hospital diagnostic laboratories are majorly expected to 
contribute to patient care and antimicrobial stewardship programs by facilitating timely selection of cost-effective antibiotic therapy and AMR surveillance, ${ }^{6}$ it is not known whether and to what extent these functions are met. We assessed the utility of AST facilities in three Ugandan hospitals.

\section{METHODS}

\section{Study design and setting}

This was a cross-sectional study. Quantitative data was collected between 27 January and 14 February 2014 from three major public referral hospitals in Uganda. Mbarara hospital is a 600-bed regional referral hospital for Western Uganda. Butabika hospital, the national mental health referral hospital in Uganda, has a 550-bed capacity. Mulago hospital is Uganda's national referral and specialist hospital, and the largest in Uganda with a 1790 bed capacity. These were three of the eight government-run hospitals offering free AST services.

Mulago, Mbarara, and Butabika hospitals had three, six, and three central laboratories respectively. Laboratory personnel at the respective hospitals were fourteen, three, and four. Each hospital had one central microbiology laboratory that used the Kirby-Bauer disk diffusion method for AST, performed according to standard operating procedures adopted from the Clinical and Laboratory Standards Institute guidelines. Laboratory personnel manually recorded and stored AST results in counter books. Notably, however, no routine AST reports or antibiograms were available at any of the hospitals during the study period.

\section{Ethics and consent}


The Makerere University school of health sciences research and ethics committee (REF 2013-033), and the Uganda National Council of Science and Technology and President's Office (HS 1554) approved the study. We obtained additional approvals from the administrative offices of the three participating hospitals.

\section{Study procedures}

We retrospectively reviewed the hospital records for November and December 2013. From the pharmacy records, we determined the antibiotic consumption, and from the microbiology records, we evaluated the AST technique used, performed tests, and analytical turnaround time. For the performed AST tests, we captured the registration and result dispatch dates, ward, and antibiotic disks used. Analytic turnaround time was defined as the time interval between specimen receipt and registration, and the issue of AST results. This interval includes time for bacterial identification, culture, and AST. Pre- (test ordering, collection, labeling, transportation) and post- (physician receipt, interpretation, and action) analytical time intervals were unavailable.

Treatment files with at least one antibiotic prescription on the in-patient wards were purposively sampled and reviewed to determine the AST request and performance rates. Data abstraction was performed using pre-tested abstraction checklists.

\section{Statistical analysis}

The data was coded, cleaned and entered in EpiData entry software v.3.1 (EpiData Association, Denmark) and Ms Excel 2013 (Microsoft Corporation, USA), and exported to STATA 14.0 (StataCorp LP,USA) for analysis. The dispensed antibiotics and 
antibiotic disks used for AST were summarized as proportions. The analytical turnaround time for AST was summarized using a median and interquartile range and used as a measure of laboratory efficiency. We determined the spearman's rank correlation between the dispensed antibiotics and the antibiotic-disks used in AST. In calculating the correlation coefficient, we combined the following antibiotics: cefotaxime and ceftriaxone; ciprofloxacin and levofloxacin; tetracycline and doxycycline; and azithromycin and erythromycin. These antibiotics have similar susceptibility data and antibiotic classification. However, amoxicillin and ampicillin were not combined because of observed disparities in susceptibility. We also performed a simple linear regression analysis to determine whether antibiotic consumption predicted AST performance.

\section{RESULTS}

Of the 607 in-patient files reviewed, 24 (4.0\%) had a documented AST request and only $13(2.1 \%)$ were performed in the three hospitals during the study period. The median AST analytical turnaround time was 5 days (IQR 4-8 days). The most widely utilized antibiotic-disks for AST were cotrimoxazole (13.0\%), ampicillin (11.7\%), and ceftriaxone (10.5\%). In contrast, healthcare providers dispensed metronidazole (30.3\%), amoxicillin $(19.6 \%)$, and ceftriaxone (14.8\%) the most (Table 1). However, there was a weak

relationship between AST disks and antibiotic use $(r=0.313, p=0.120)$ as shown in Figure 1. There was no association between antibiotic consumption and AST performance from the regression model (results not shown).

\section{DISCUSSION}


The study showed very low utilization of AST where the proportion of AST requests was $4 \%$, and only $2 \%$ were performed. This low AST performance is similar to that reported in Ethiopia (3.8\%) while vastly divergent from that reported among six US hospitals $(59 \%)^{7,8}$. Uganda, like Ethiopia, is a resource-limited country that lacks basic diagnostic resources required for $\mathrm{AST}^{5,9}$. The Kirby-Bauer disk diffusion method used at the study sites costs about 2.5-5 USD per test and requires overnight incubation for $16-24 \mathrm{~h}{ }^{10}$. The alternative AST methods such as MicroScan Walk-Away (Siemens Healthcare Diagnostics) cost about 5.50-6.35 USD per panel and results can be read in as short as 3.5 hours ${ }^{10,11}$. Even though most RLS have opted for the inexpensive disk method, the automated systems are considered more cost-effective due to their reduced turnaround time and increased efficiency ${ }^{12,13}$. Additionally, automated methods are more clinically relevant in providing prompt guidance to antimicrobial use.

Given that the median length of stay in Ugandan hospitals is 4.2 days, patients who stay for less than 5 days are unlikely to receive their AST results before discharge ${ }^{14}$. The need for bacterial cultures that take 24-48 hours or more, the high workload, and lack of mechanization with the disk diffusion method contribute to the long turnaround times observed ${ }^{5,15,16}$. The workload in public hospital laboratories in Uganda is high because they serve high patient numbers, yet there are few experienced personnel ${ }^{5}$, with staffing levels less than $38 \%$ of the required ${ }^{9}$. Notably, even when the AST request rate is only about $4 \%$, only about half of the requests are processed - this may have demotivated physicians from requesting for AST. It is clear, however, that deliberate measures to improve laboratory efficiency and AST capacity as part of hospital antimicrobial stewardship programs are necessary. With improved laboratory 
efficiency, AMR associated mortality, hospital delays, and cost, and also antibiotic misuse will decrease simply because physicians can access the results faster and administer effective antibiotic therapy ${ }^{17,18}$. RLS can achieve improved laboratory efficiency and reduced AST turnaround time through increased staffing and investment in diagnostics, and also through the use of satellite laboratories and automated AST systems ${ }^{19}$.

The discrepancy between antibiotic consumption and AST performance rate is highlighted by the weak correlation and lack of linear association. This discrepancy may indicate poor coordination in the procurement of drugs and microbiology reagents in RLS $^{5}$. Since AST performance should mirror the antibiotic prescribing patterns and the available antibiotics in the hospitals ${ }^{6,10,20,21}$, it is doubtful that the physicians prescribe effective antibiotics based on local resistance patterns. Therefore, hospitals need to bear in mind their antibiotic consumption when sourcing AST reagents such that prevailing AMR is effectively detected and reported.

This study, however, did not standardize the antibiotic consumption as defined by daily doses or dispensed daily doses. Additionally, the three hospitals studied may not geographically represent the entire country or practices in the other eight hospitals were AST was practiced. Selection bias may have been introduced in the selection of hospitals, in-patients, or hospital wards, and this may affect the generalizability of study results. However, we recommend future works comparing the cost-effectiveness and clinical impact of the disk diffusion method with alternative AST methods, and the development of other low-cost AMR diagnostics suitable for RLS such as point-of-care tests. 


\section{CONCLUSION}

We report underutilization of AST in three referral hospitals in Uganda and a discrepancy between AST requests and dispensed antibiotics. This underutilization may be due to the commonly used Kirby-Bauer disk diffusion method in hospitals of RLS. The disk diffusion method is has the longest turnaround time. Alternative highthroughput AST technologies that are not overly dependent on laboratory personnel may be more cost-effective and have the potential of increasing AST utility. To improve AMR surveillance, hospitals in RLS should also consider procuring AST reagents based on their local antibiotic consumption.

\section{Acknowledgements}

The authors thank Emmy Enyiku, Lawrence Otim, Andrew Basenero, Patricia Nabayego, and Merab Babirye for collecting the data and Alan Kinene for analyzing the study data. The authors also acknowledge the staff of Mulago, Mbarara, and Butabika hospitals for enabling them to collect data from their respective hospitals.

\section{Author contributions}

I.S conceived and designed the study; collected, analyzed, and interpreted the data; drafted the manuscript; takes responsibility for the integrity and accuracy of the data and analysis; and is accountable for all aspects of the work. K.S and D.K interpreted the data and reviewed the manuscript critically for important intellectual content. J.M conceived the study; critically reviewed the manuscript; offered administrative, technical and material support; and supervised the study. All authors have read and approved the final version of the manuscript submitted for publication. 


\section{Disclosure statement}

The authors report no conflicts of interest.

\section{Funding information}

This work was supported by the Medical Education for Equitable Services to All Ugandans and Medical Education Partnership Initiative (grant number, 5R24TW008886), the Office of Global AIDS Coordinator, and the U. S. Department of Health and Human Services, Health Resources and Services Administration and National Institutes of Health. The funders played no role in the design of the study, collection of data, analysis and interpretation of data, writing of the report or submission of this manuscript for publication.

\section{REFERENCES}

1. World Health Organization. Global Action Plan on Antimicrobial Resistance. (2015).

2. O'Neill, J. Review on Antimicrobial Resistance: Tackling a crisis for the health and wealth of nations. (2014).

3. Liz, T. Antimicrobial Resistance: Global Burden. https://www.who.int/medicines/technical_briefing/tbs/TBS2016_AMR_GAP.pdf (2016).

4. Brown, R. C. Antibiotic sensitivity testing for infections in developing countries: lacking the basics. JAMA vol. 276 952-953 (1996).

5. UNAS, CDDEP, GARP-Uganda, Mpairwe, Y., \& Wamala, S. Antibiotic Resistance in Uganda: Situation Analysis and Recommendations Antibiotic Resistance in Uganda: Situation Analysis and Recommendations UGANDA NATIONAL ACADEMY OF 
SCIENCES. (Uganda National Academy of Sciences; Center for Disease Dynamics, Economics \& Policy, 2015).

6. Morency-Potvin, P., Schwartz, D. N. \& Weinstein, R. A. Antimicrobial Stewardship: How the Microbiology Laboratory Can Right the Ship. Clin. Microbiol. Rev. 30, 381-407 (2017).

7. Gutema, G., Hakonsen, H., Engidawork, E. \& Toverud, E.-L. Multiple challenges of antibiotic use in a large hospital in Ethiopia - a ward-specific study showing high rates of hospital-acquired infections and ineffective prophylaxis. BMC Health Serv. Res. 18, 326 (2018).

8. Braykov, N. P. et al. Assessment of empirical antibiotic therapy optimisation in six hospitals: an observational cohort study. Lancet. Infect. Dis. 14, 1220-1227 (2014).

9. Ministry of Health Uganda. Uganda National Health Laboratory Services Policy. http://apps.who.int/medicinedocs/documents/s22320en/s22320en.pdf (2009).

10. Reller, L. B., Weinstein, M., Jorgensen, J. H. \& Ferraro, M. J. Antimicrobial Susceptibility Testing: A Review of General Principles and Contemporary Practices. Clin. Infect. Dis. 49, 1749-1755 (2009).

11. Siemens Healthcare Diagnostics. MicroScan ® Microbiology Systems Siemens Healthcare Diagnostics Department of Veterans Affairs Federal Supply Schedule ( FSS ) Cost-Per-Test Program MicroScan ® Microbiology Systems. (2011).

12. Snyder, J. W., Munier, G. K. \& Johnson, C. L. Direct Comparison of the BD Phoenix System with the MicroScan WalkAway System for Identification and Antimicrobial Susceptibility Testing of \&lt;em\&gt;Enterobacteriaceae\&lt;/em\&gt; and Nonfermentative Gram-Negative Organisms. J. Clin. Microbiol. 46, 2327 LP - 2333 (2008). 
13. Pliakos, E. E., Andreatos, N., Shehadeh, F., Ziakas, P. D. \& Mylonakis, E. The CostEffectiveness of Rapid Diagnostic Testing for the Diagnosis of Bloodstream Infections with or without Antimicrobial Stewardship. Clin. Microbiol. Rev. 31, e00095-17 (2018).

14. Republic of Uganda Ministry of Health. Uganda Hospital and Health Centre IV Census Survey. WHO/MoH collaborative report. (2014).

15. Miller, J. M. et al. A Guide to Utilization of the Microbiology Laboratory for Diagnosis of Infectious Diseases: 2018 Update by the Infectious Diseases Society of America and the American Society for Microbiology. Clin. Infect. Dis. 67, 813-816 (2018).

16. VKE Lim and YM Cheong. Bacteriology turnaround time in seven Malaysian general hospitals. Malays. J. Pathol. 14, 41-43 (1992).

17. Kerremans, J. J. et al. Rapid identification and antimicrobial susceptibility testing reduce antibiotic use and accelerate pathogen-directed antibiotic use. J. Antimicrob. Chemother. $61,428-435$ (2008).

18. Barenfanger, J., Drake, C. \& Kacich, G. Clinical and financial benefits of rapid bacterial identification and antimicrobial susceptibility testing. J. Clin. Microbiol. 37, 1415-1418 (1999).

19. Hawkins, R. C. Laboratory turnaround time. Clin. Biochem. Rev. 28, 179-194 (2007).

20. Jorgensen, J. H. Selection of antimicrobial agents for routine testing in a clinical microbiology laboratory. Diagn. Microbiol. Infect. Dis. 16, 245-249 (1993).

21. Performance Standards for Antimicrobial Susceptibility Testing; Twenty-Second Informational Supplement. vol. 32 (2012). 


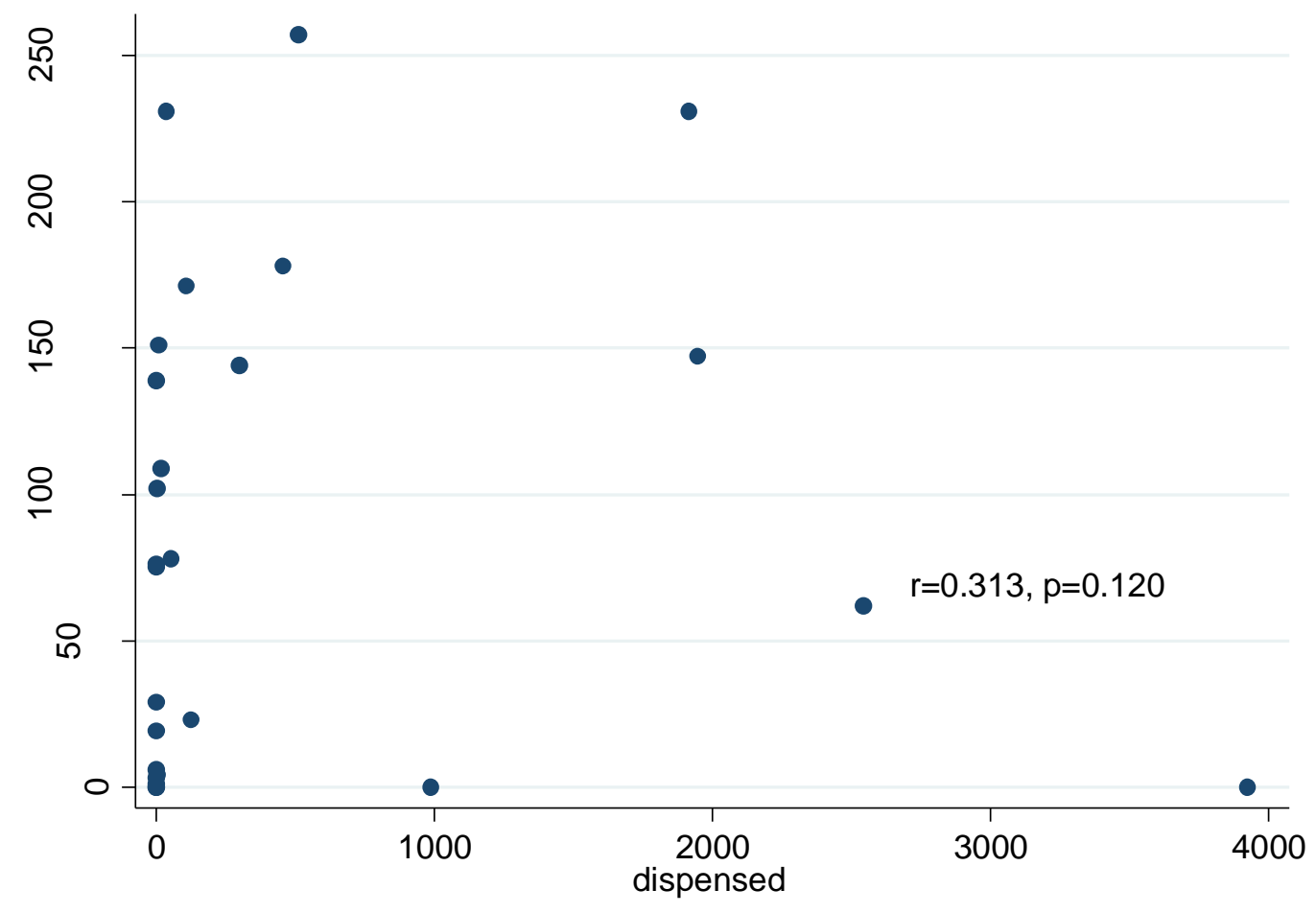

Figure 1: Scatter plot for dispensed antibiotics and AST antibiotic disks 
Table 1: Antibiotics dispensed and AST disks in the labs of the three hospitals

\begin{tabular}{|c|c|c|c|c|c|c|c|}
\hline \multicolumn{6}{|c|}{ Most dispensed antibiotics } & \multicolumn{2}{|c|}{ Laboratory AST } \\
\hline Antibiotics & Mulago & Mbarara & Butabika & Total & $\%$ & Freq. $^{*}$ & $\%$ \\
\hline Metronidazole & 1,462 & 1,665 & 799 & 3,926 & 30.3 & 0 & 0.0 \\
\hline Amoxicillin & 366 & 1,634 & 545 & 2,545 & 19.6 & 62 & 3.1 \\
\hline Ceftriaxone & 1,917 & 0 & 0 & 1,917 & 14.8 & 207 & 10.5 \\
\hline Ciprofloxacin & 674 & 912 & 233 & 1819 & 14.0 & 147 & 7.4 \\
\hline Ampiclox & 313 & 674 & 1 & 988 & 7.6 & 0 & 0.0 \\
\hline Cotrimoxazole & 78 & 182 & 251 & 511 & 3.9 & 257 & 13.0 \\
\hline Doxycycline & 65 & 392 & 0 & 457 & 3.5 & 0 & 0.0 \\
\hline Erythromycin & 146 & 151 & 0 & 297 & 2.3 & 144 & 7.3 \\
\hline Levofloxacin & 131 & 0 & 0 & 131 & 1.0 & 0 & 0.0 \\
\hline Cloxacillin & 95 & 31 & 0 & 126 & 1.0 & 23 & 1.2 \\
\hline Gentamicin & 109 & 0 & 0 & 109 & 0.8 & 171 & 8.7 \\
\hline Cefuroxime & 54 & 0 & 0 & 54 & 0.4 & 78 & 3.9 \\
\hline Ampicillin & 38 & 0 & 0 & 38 & 0.3 & 231 & 11.7 \\
\hline Co-Amoxiclav & 17 & 0 & 0 & 17 & 0.1 & 109 & 5.5 \\
\hline Chloramphenicol & 0 & 8 & 0 & 8 & 0.1 & 151 & 7.6 \\
\hline Others & 7 & 2 & 0 & 9 & $<0.1$ & 656 & 33.2 \\
\hline Total & 5,651 & 1,829 & 5,472 & 12,952 & $100 \%$ & - & - \\
\hline
\end{tabular}

